Katharina Wulf*, Michael Teske, Daniela Arbeiter, Thomas Eickner, Gerrit Paasche, Thomas Lenarz and Niels Grabow

\title{
Covalent coupling of polymers as drug delivery coatings on silicone surfaces for future applications
}

\begin{abstract}
Insertion of a cochlear implant (CI) into the scala tympani evokes electrode insertion trauma that can result in mechanical damage of the lateral and medial wall and causes ingrowth of fibrous tissue on the implant surface. In consequence, it is of great clinical interest to modify the silicone surface of $\mathrm{CI}$ electrodes in order to inhibit and minimize the overgrowth by connective tissue.

In this case, the covalent coupling of bifunctional polymers on common CI silicone surfaces is a versatile method to influence the implant cell interaction, but is still a challenge due to the chemically unreactive properties of silicone.

We present a sophisticated method to apply a biodegradable polymer coating, such as polylactide or polyhydroxybutyrate, on silicone surfaces to generate a drug delivery system using a covalently bound intermediate layer. The adhesive coatings were applied via spincoating processes with different adherence to the intermediate layer.

Changes in surface morphology were investigated by comparison of pure silicone and coated silicone. Specific polymer bands of the infrared spectra were detected by IR spectroscopy. Results indicate a complete coating with polylactide or polyhydroxybutyrate. The presented method is a versatile tool for the coating of silicone CI electrodes with degradable polymer layers.
\end{abstract}

Keywords: silicone, PLLA, P(3HB), covalent coupling, FTIR-ATR, melting temperature, adhesion.

https://doi.org/10.1515/cdbme-2019-0078

*Corresponding author: Katharina Wulf Institute for Biomedical Engineering, Rostock University Medical Center Rostock, Germany, katharina.wulf@uni-rostock.de

Michael Teske, Daniela Arbeiter, Thomas Eickner, Niels Grabow: Institute for Biomedical Engineering, Rostock University Medical Center, Rostock, Germany

Gerrit Paasche, Thomas Lenarz: Department of Otorhinolaryngology, Head and Neck Surgery, Hanover Medical School, Hanover, Germany

\section{Introduction}

Silicone is cost effective, biocompatible, non-toxic and exhibits excellent optical properties. It is used in medical applications, such as lenses and cochlear implant (CI) electrodes [1]. However, the insertion of the CI into the scala tympani evokes electrode insertion trauma resulting in mechanical damage of the lateral and medial wall and, most adversely, in growth of fibrous tissue on the electrode. Thus, not only the impedance at the electrode-tissue interface is increased and higher power is needed to ensure CI performance, also selective neuronal stimulation for discrimination between different sound frequencies is disturbed. A promising approach to inhibit and minimize the overgrowth by connective tissue is to coat the silicone surface with polymers which can act as a drug delivery system for antifibrotic drugs. However, the modification of silicone surfaces is still a challenge due to the inert properties, their low surface energy and the resulting poor adhesion of the polymeric coatings [2].

Based on previous studies [3] we investigated the binding of biodegradable, biocompatible and suitable for drug release, polymers poly-L-lactide (PLLA) or poly(3hydroxybutyrate) $(\mathrm{P}(3 \mathrm{HB})$ to silicone via a covalently coupled intermediate layer. In this case poly-L-lysine, as intermediate layer was covalently coupled to oxygen $\left(\mathrm{O}_{2}\right)$ plasma activated silicone surface via (3glycidyloxypropyl)trimethoxysilane (GOPS) followed by spin coating of PLLA or P(3HB).

The here described coated silicone surface can decisively influence the implant-cell interaction by acting as a drug delivery system. 


\section{Materials and Methods}

\subsection{Materials}

The silicone slides (Sil) were provided by MED-EL (Innsbruck, Austria). Poly-L-lactide (PLLA, L210) was purchased from Evonik (Schwerte, Germany) and the polyhydroxy butyric acid derivate $(\mathrm{P}(3 \mathrm{HB})$ $(\mathrm{Mw}=850.000 \mathrm{~g} / \mathrm{mol})$ was provided by the HelmholtzZentrum für Umweltforschung, Leipzig, Germany. The crosslinker (3-glycidyloxypropyl)trimethoxysilane (GOPS) and Poly-L-Lysine (PLL) were purchased from Sigma Aldrich, Munich, Germany.

\subsection{Methods}

The cleaned silicone samples were activated via $\mathrm{O}_{2}$ plasma using $100 \mathrm{~W}$ power at $0.3 \mathrm{mbar}$ for $1 \mathrm{~min}$ in a plasma chamber (Diener, Ebhausen, Germany). Then the samples were incubated in pure GOPS for $6 \mathrm{~h}$ at $90^{\circ} \mathrm{C}$. The activated samples were rinsed 3 times with ethanol and dried at $80^{\circ} \mathrm{C}$ overnight under vacuum at 40 mbar. The resulting dried samples were spin coated with the specific polymer using a spin coater (SCE-150, LOT-QuantumDesign GmbH, Darmstadt, Germany). Therefore, several drops of the polymer solution $(1 \mathrm{wt} \%)$ were placed onto the silicone slide and the chuck was rotated for $2 \mathrm{~min}$ at $200 \mathrm{rpm}$. The coated slides were dried under vacuum overnight.

The surfaces were examined in a XL30 (Philips, Eindhoven, The Netherlands) scanning electron microscope (SEM). A Contact Angle System (OCA 20, Dataphysics Instruments GmbH, Filderstadt, Germany) was used for analyzing surface modifications by contact angle measurements of ultra-pure water sessile drops. Presented mean values and standard deviations were calculated from $\mathrm{n}=5$ samples.

FTIR-ATR-measurements of the investigated silicone samples were performed using a Bruker Vertex 70 IRspectrometer (Bruker, Ettlingen, Germany) equipped with a DLaTGS-detector. Each spectrum has been achieved in the range of $4000-500 \mathrm{~cm}^{-1}$ at a spectral resolution of $4 \mathrm{~cm}^{-1}$ and with 32 scans on the average using a Graseby Golden Gate Diamond ATR-unit. All spectra were analysed using OPUS software (Bruker, Ettlingen, Germany) and were subsequently atmosphere and baseline corrected.

Thermal analysis was carried out with a DSC1 (Mettler Toledo GmbH, Greifensee, Switzerland) under a nitrogen purge. High purity indium was used for temperature and heat of fusion $(\Delta \mathrm{H})$ calibration. The scans of $n=3$ films per subgroup were performed from -90 to $220^{\circ} \mathrm{C}$ at a heating rate of $20^{\circ} \mathrm{C} / \mathrm{min}$. The data were analyzed with respect to the melting temperature $\left(T_{m}\right)$.

Preliminary tests of the detachment of the specific polymer coatings from the silicone were analysed via SEM. Therefore, the silicone slides were incubated in a phosphate buffered saline solution (PBS, $\mathrm{pH}$ 7.6) for at least $24 \mathrm{~h}$ at $37^{\circ} \mathrm{C}$.

\section{Results}

In this investigation the coating of Sil with biodegradable polymers using GOPS as crosslinker and PLL as intermediate layer were investigated. The different coatings were produced via spin coating.

The surface morphology of the silicone surface is changed after each deposition. The surface got more structured with addition of GOPS and PLL and the polymer coating. The $\mathrm{P}(3 \mathrm{HB})$ surface seems to be more structured compared to the PLLA coated samples (Fig. 1).
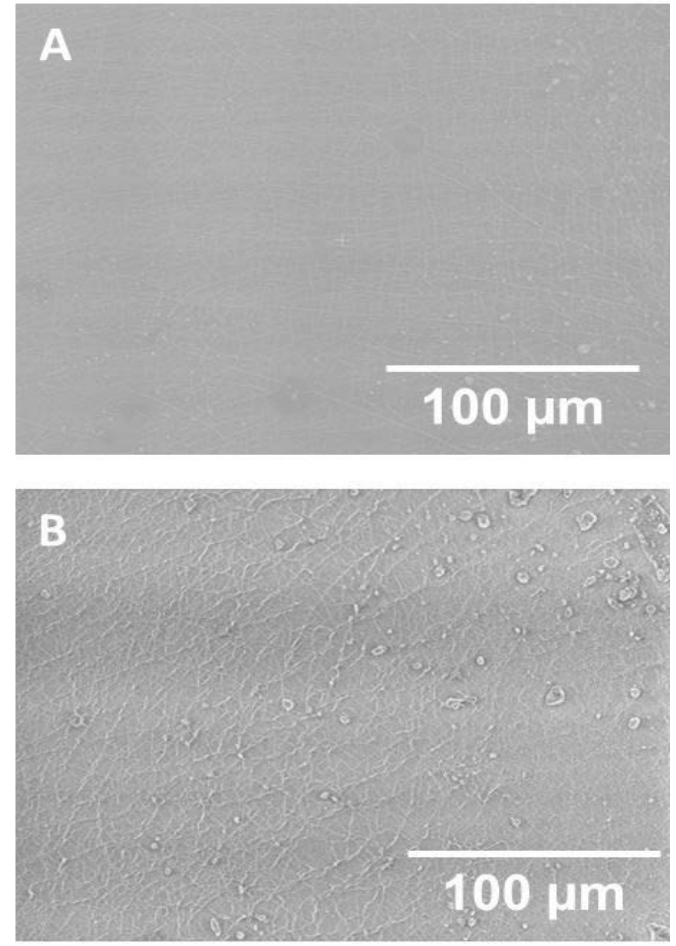

Figure 1: Representative SEM images of polymer coated silicon samples (A) Sil_GOPS_PLL_PLLA, (B) Sil_GOPS_PLL_P(3HB).

After each step the contact angle changed (Tab. 1). Furthermore, the contact angle for PLLA and P(3HB) are rather similar to specific reference coatings (PLLA or $\mathrm{P}(3 \mathrm{HB})$ coated glass slides). 
Table 1: Water contact angle $\Theta_{w} \pm$ standard deviation (SD) on several silicone surfaces for sessile drop method $(n=5)$.

\begin{tabular}{ll}
\hline polymer & Contact angle $\left[^{\circ}\right]$ \\
\hline Sil & $129.6 \pm 4.1$ \\
Sil_GOPS & $83.0 \pm 4.3$ \\
Sil_GOPS-PLL & $98.7 \pm 2.4$ \\
Sil_GOPS-PLL-PLLA & $133.6 \pm 2.5$ \\
Sil_GOPS-PLL-P(3HB) & $110.8 \pm 1.0$ \\
\hline
\end{tabular}

Melting temperature of the investigated silicone was determined following differential scanning calorimetry (DSC). For all polymer coated silicones, a $T_{m}$ around $180{ }^{\circ} \mathrm{C}$ aroused. The $T_{m}$ is for all samples rather similar due to the pure coating polymers, PLLA, $\mathrm{P}(3 \mathrm{HB})[4,5]$ as shown in table 2 .

Table 2: Melting Temperature $T_{m} \pm$ standard deviation (SD) of coated silicone surfaces and their specific references $(n=3)$, * literature data $[4,5]$.

\begin{tabular}{ll}
\hline polymer & $\boldsymbol{T}_{\boldsymbol{m}} \pm \mathrm{SD}\left[^{\circ}{ }^{ }\right]$ \\
\hline Sil & - \\
Sil_GOPS-PLL-PLLA & $175.4 \pm 2.8$ \\
PLLA $^{*}$ & $178.7 \pm 0.2$ \\
Sil_GOPS-PLL-PHB1 $^{*}$ & $172.1 \pm 2.2$ \\
PHB1* $^{*}$ & 178 \\
\hline
\end{tabular}

Furthermore, the changes in IR bands indicate a chemical change of the surface composition. Differences of

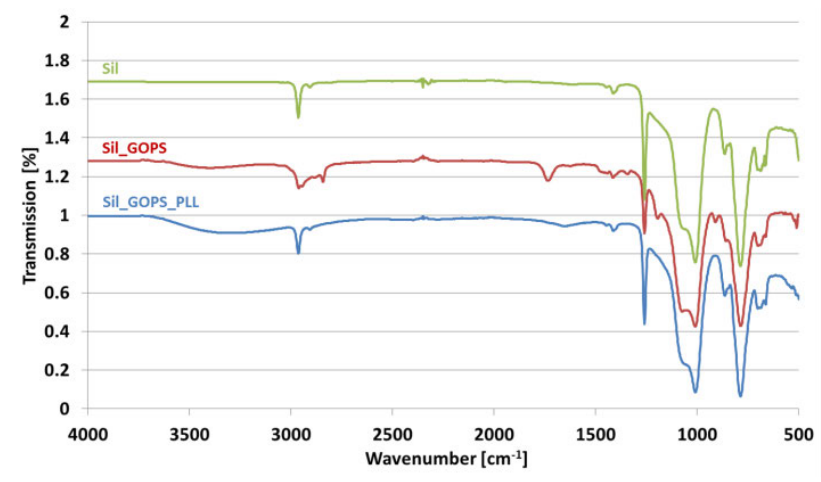

Figure 2: FTIR-ATR spectra of the modified silicon samples Sil, Sil_GOPS and Sil_GOPS_PLL, in the range of $4000-500 \mathrm{~cm}^{-1}$.

the IR bands in the range of $3500-3000 \mathrm{~cm}^{-1}$ and $1800-$ $1700 \mathrm{~cm}^{-1}$ can be seen comparing the IR spectra Sil, Sil_GOPS and Sil_GOPS_PLL (Fig. 2).
All Sil_GOPS_PLL_polymer samples exhibit several characteristic bands of the specific polymer as well as bands specific for the coated silicone (Fig. 3). This was most obvious for the PLLA coated samples. The characteristic IR bands for the PLLA samples are more distinct than for $\mathrm{P}(3 \mathrm{HB})$. IR spectra for all polymer coated silicone samples reveal a prominent band between $1764-1712 \mathrm{~cm}^{-1}$ (Fig. 3), which does not appear in the other IR spectra (Fig. 2). This band denotes carbonyl $(\mathrm{C}=\mathrm{O})$ vibration, characteristic for ester bonds found in the used coating polymers.

Preliminary peel tests showed that the PLLA interacts relatively strongly with the modified silicone samples.
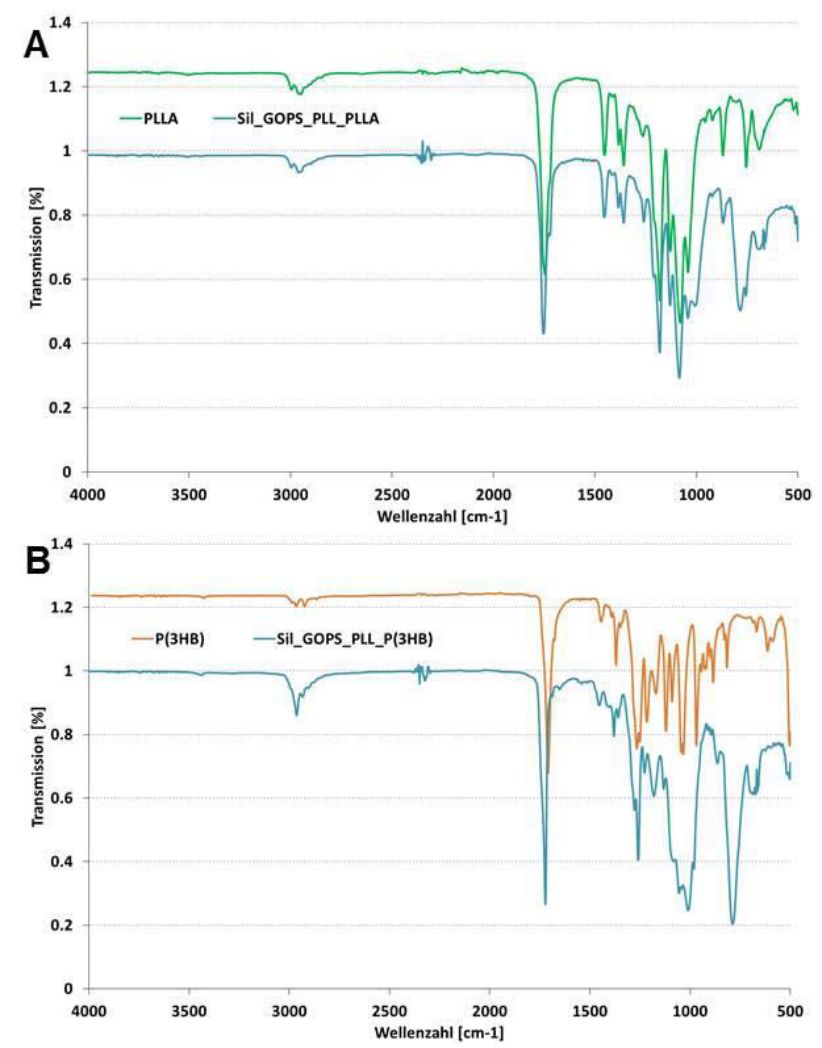

Figure 3: FTIR-ATR spectra of the polymer coated silicon samples (A) PLLA and Sil_GOPS_PLL_PLLA, (B) P(3HB) and Sil_GOPS_PLL_P(3HB) in the range of $4000-500 \mathrm{~cm}^{-1}$.

$\mathrm{P}(3 \mathrm{HB})$ could be peeled of the silicone in PBS buffer already after 24 hours of incubation.

\section{Discussion}

Due to the inert properties of silicone, bonding or coating with polymers is a challenge, but necessary for various medical applications to create optimal conditions.

In order to obtain an adhesive polymer coating, a covalently coupled intermediate layer was developed with 
which the selected biodegradable polymers could interact. As intermediate layer GOPS as crosslinker and PLL were examined.

The contact angle measurements indicate a successful coating of the silicone samples. After each reaction step the contact angle is changed. Furthermore, the contact angles of the coated samples are similar to their reference.

The appearance of the ancillary melting temperature confirms the successful coating of the silicone. Moreover, the similarity of these melting temperatures to the pure polymers used for the coating also supports this assumption. Additionally, the changes in IR spectra indicate a chemical change of the surfaces. IR spectra of the polymer coated samples exhibit several characteristic bands of the pure polymer as well as bands that correspond to the pure silicone [6].

The first peel tests performed confirm that PLLA interact well with the intermediate layer modified silicone. $\mathrm{P}(3 \mathrm{HB})$ appears to have a relatively weak interaction, as it quickly detaches from the silicone.

In summary, with regard to medical applications, such as an antifibrotic drug loaded coating of CI electrodes, the coating methods are promising. The study described here shows the successful covalent coupling of an intermediate layer to silicone and the following coating with biodegradable polymers, which have already been tested as drug delivery systems [7]. Initial adhesion studies have shown a relative stable interaction between the intermediate layer and PLLA. However, insertion experiments of model electrodes for CI, coated with the presented method, are conducted to further evaluate the adhesion of the coating.

Acknowledgment: The authors would like to thank Andrea Rohde, Caroline Dudda, Martina Nerger, Katja Hahn and Babette Hummel for the technical assistance.

\section{Author's Statement}

Research funding: Partial financial support by the European Regional Development Fund (ERDF) and the European Social Fund (ESF) within the collaborative research between economy and science of the state Mecklenburg-Vorpommern and by the Federal Ministry of
Education and Research (BMBF) within RESPONSE "Partnership for Innovation in Implant Technology" is gratefully acknowledged.

Conflict of interest: Authors state no conflict of interest. Informed consent: Informed consent has been obtained from all individuals included in this study.

\section{References}

[1] Curtis J, Colas A. Medical Applications of Silicones. In: Ratner BD, editor. Biomaterials science: An introduction to materials in medicine. 3rd ed. Amsterdam, Heidelberg: Elsevier; 2013:1106-1116. doi:10.1016/B978-0-08-0877808.00107-8.

[2] Rahimi A, Mashak A. Review on rubbers in medicine: Natural, silicone and polyurethane rubbers. Plastics, Rubber and Composites. 2013;42:223-30. doi:10.1179/1743289811Y.0000000063.

[3] Bohl A, Rohm HW, Ceschi P, Paasche G, Hahn A, Barcikowski S, et al. Development of a specially tailored local drug delivery system for the prevention of fibrosis after insertion of cochlear implants into the inner ear. J Mater Sci Mater Med. 2012;23:2151-62. doi:10.1007/s10856-0124698-z.

[4] Blümm E, Owen AJ. Miscibility, crystallization and melting of poly(3-hydroxybutyrate)/ poly(l-lactide) blends. Polymer. 1995;36:4077-81. doi:10.1016/0032-3861(95)90987-D.

[5] Arbeiter D, Kohse S, Eickner T, Grabow N, Schmitz K-P. Influence of additives on physico-chemical properties of electrospun poly(L-lactide). Current Directions in Biomedical Engineering. 2018;4:493-6. doi:10.1515/cdbme-2018-0118.

[6] Bayarı S, Severcan F. FTIR study of biodegradable biopolymers: $\mathrm{P}(3 \mathrm{HB}), \mathrm{P}(3 \mathrm{HB}-\mathrm{co}-4 \mathrm{HB})$ and $\mathrm{P}(3 \mathrm{HB}-\mathrm{co}-3 \mathrm{HV})$. Journal of Molecular Structure. 2005;744-747:529-34. doi:10.1016/j.molstruc.2004.12.029.

[7] Löbler M, Sternberg K, Stachs O, Allemann R, Grabow N, Roock $A$, et al. Polymers and drugs suitable for the development of a drug delivery drainage system in glaucoma surgery. J Biomed Mater Res Part B Appl Biomater. 2011;97:388-95. doi:10.1002/jbm.b.31826. 